\title{
Variance-Optimal Hedging for the Process Based on Non-Extensive Statistical Mechanics and Poisson Jumps
}

\author{
PAN ZHAO ${ }^{a, b, c, *}$ AND QINGXIAN XIAO ${ }^{a}$ \\ ${ }^{a}$ Business School, University of Shanghai for Science and Technology, Shanghai, China \\ ${ }^{b}$ College of Finance and Mathematics, West Anhui University, Lu'an, Anhui, China \\ ${ }^{c}$ Financial Risk Intelligent Control and Prevention Institute of West Anhui University, Lu'an, Anhui, China \\ (Received December 30, 2015; revised version March 28, 2016; in final form April 4, 2016)

\begin{abstract}
In this study, we consider a minimum-variance hedging problem in an incomplete market, in which the risky
\end{abstract} \\ asset is driven by the process based on non-extensive statistical mechanics and Poisson jumps. Using the stochastic \\ control theory and backward stochastic differential equation method, we obtain a closed-form solution for the \\ minimum-variance hedging policy.
}

DOI: 10.12693/APhysPolA.129.1252

PACS/topics: 89.65.Gh

\section{Introduction}

We know that it is very important for investors to accurately describe the law of asset price movement. The asset price model based on Brownian motion has appeared in many literatures. For example, Black and Scholes (B-S) [1] and Merton [2] studied the prices of financial derivatives of the underlying stock following such a model. It is well known that the hypothesis that the asset price changes follow Brownian motion implies that the price changes are independent and the distribution of log-returns is normal. However, several studies have shown that the distribution of empirical logreturns does not follow the normal distribution. A number of econophysics scholars have found that asset returns have a long-term memory characteristic in general [3-5] and the distribution of returns usually exhibits a fat-tail phenomenon [6-8].

In 1988, Tsallis generalized the Boltzmann-Gibbs statistics and proposed a non-extensive statistical approach [9]. As the development of the Tsallis nonextensive statistics, several econophysics researchers have applied the power-law distribution characteristic of the Tsallis statistics framework to describe the fat-tail phenomenon of asset returns [10-14]. Moreover, Borland $[15,16]$ proposed an asset price model in which the driving noise followed a generalized Brownian motion governed by a Tsallis distribution of index $q$. For $q>1$, the Tsallis distribution exhibits fat tails. For $q=1$, it coincides with a normal distribution and the standard $\mathrm{B}-\mathrm{S}$ model is recovered. However, in reality the distribution of asset returns is generally asymmetric and has significant skewness [17-19]. Therefore, the symmetric Tsallis distribution has certain shortcomings in modeling the skew distribution of returns. Moreover, in the field of

*corresponding author; e-mail: zhaopan1980@163.com finance, the jump process is generally employed to control the skewness of the distribution of returns [20,21].

In this paper, to accurately describe asset price processes, we consider the fat-tail and skew phenomena of the distribution of returns and propose an asset price model with a Tsallis distribution of index $q$ and the Poisson jump, which can describe the characteristics of long-run dependence of asset prices and the skewness of the distribution of returns. Furthermore, the minimumvariance hedging problem under this model is studied. The optimal hedging problem is always an important issue in the financial field. Lim [22] and Lim and Zhou [23], using backward stochastic differential equations, studied the problem under the assumption that the asset price process was continuous and driven by the Brownian motion. Using the convex duality and projection Theorem, Gourieroux [24], Laurent and Pham [25], and Schweizer [26] studied that under the assumption that the asset price process was continuous semi-martingale. However, the literatures on the asset price model with discontinuous processes are relatively few. Moreover, in this paper, the market is incomplete. The methods used to solve a minimum-variance hedging problem of complete markets are not valid. We therefore use the stochastic control theory and backward stochastic differential equations to solve this problem. Finally, a closedform solution for the minimum-variance hedging policy is obtained.

This paper is organized as follows. In Sect. 2, we model a price process in which the risky asset is described by the Tsallis non-extensive statistical mechanics and Poisson jumps. Then, we formulate the minimum-variance hedging problem as a stochastic control problem. In Sect. 3 we derive the minimum-variance hedging strategy by the use of the stochastic control theory and find that the optimal hedging portfolio for our model depends on the solution of a backward stochastic differential equation. In the final section, we summarize the paper. 


\section{Market model and optimal hedging problem}

Suppose that there are two types of tradable assets in the financial market. One is a risk-free asset, called a bond. The price $B(t)$ of the bond follows the equation:

$$
\mathrm{d} B(t)=r B(t) \mathrm{d} t ; \quad B(0)=1,
$$

where $r$ is the risk-free interest rate. The other is a risky asset, called a stock. Its price $S(t)$ satisfies the stochastic differential equation

$$
\begin{aligned}
& \mathrm{d} S(t)=\mu S(t) \mathrm{d} t+\sigma S(t) \mathrm{d} \Omega(t)+u(t) S(t) \mathrm{d} N(t) ; \\
& S(0)=S_{0},
\end{aligned}
$$

where

$$
\mathrm{d} \Omega(t)=P^{(1-q) / 2}(\Omega(t)) \mathrm{d} W(t) .
$$

The probability density function $P(\Omega(t))$ is given by $q$-Gaussian distribution derived from the Tsallis nonextensive statistics [27]. It can be written as

$$
\begin{aligned}
& P(\Omega(t), t)=\frac{1}{z(t)}\left[1-\beta(t)(1-q) \Omega(t)^{2}\right]^{1 /(1-q)}, \\
& z(t)=\int_{-\infty}^{+\infty}\left[1-(1-q) \beta(t) \Omega(t)^{2}\right]^{1 /(1-q)} \mathrm{d} \Omega(t)= \\
& \quad[(2-q)(3-q) c t]^{1 /(3-q)}, \\
& \beta(t)=c^{(1-q) /(3-q)}[(2-q)(3-q) t]^{2 /(q-3)} ; 1<q<\frac{5}{3}, \\
& c=\frac{\pi}{q-1} \frac{\Gamma^{2}\left(1 /(q-1)-\frac{1}{2}\right)}{\Gamma^{2}(1 /(q-1))}
\end{aligned}
$$

$\Gamma(\cdot)$ is a gamma function. $W(t)$ is a standard Brownian motion. $N(t)$ is a stochastic Poisson process with the intensity $\lambda . u(t)$ is the relative change in the price $S(t)$, which represents jump sizes of the prices. Let $(\Omega, \mathcal{F}, \mathcal{P})$ be a complete probability space. Assume that $W(t)$ and $N(t)$ are mutual independent. Denote $\left\{\mathcal{F}_{t}\right\}_{t \geq 0}$ is the natural filtration generated by $W(t)$, $\left\{\mathcal{N}_{t}\right\}_{t>0}$ is the filtration generated by $N(t)$, and the filtration $\mathcal{G}_{t}=\mathcal{F}_{t} \vee \mathcal{N}_{t}$ represents the information available to the investor at the time $t$. Let $H \in L^{\infty}\left(\mathcal{G}_{t}\right)$ be a random variable, which represents some liability that the investor faces in the financial market. Let $M(t)=$ $N(t)-\lambda t$ be the compensated Poisson process, which is a $\mathcal{G}_{t}$-martingale (see Lemma 6.6.3 in [28]). Then, the price process of the risky asset can be written

$$
\begin{aligned}
& \mathrm{d} S(t)=(\mu+\lambda u(t)) S(t) \mathrm{d} t \\
& \quad+\sigma P(\Omega(t))^{(1-q) / 2} S(t) \mathrm{d} W(t)+u(t) S(t) \mathrm{d} M(t) .
\end{aligned}
$$

Assume that the financial market is frictionless, and the investor can trade continuously over a finite time horizon $[0, T]$. Let $\left\{\pi_{t}\right\}_{0<t<T}$ denote the amount of wealth allocated to the risky asset at time $t .\left\{\pi_{t}\right\}_{0<t<T}$ is $\mathcal{G}_{t^{-}}$ predictable and $\int_{0}^{t} \pi_{t} d t<\infty$. Then, the total wealth process $X(t)$ is

$$
X(t)=\pi_{t} S(t)+\left(1-\pi_{t}\right) B(t) .
$$

Suppose that the portfolio $\pi_{t}$ remains unchanged over the small time interval $[t, t+\Delta t)$, which is reasonable. It is because that in reality the investor can only adjust his strategies discretely over time and the strategy decision is made at the beginning of the time horizon [29]. Thus, when the $\Delta t$ is sufficiently small, the change in wealth over the small time interval $[t, t+\Delta t)$ is

$$
\mathrm{d} X(t)=\pi_{t} \mathrm{~d} S(t)+\left(1-\pi_{t}\right) \mathrm{d} B(t) .
$$

Substituting (1) and (3) into (5), we can obtain that the wealth $X(t)$ satisfies the following stochastic differential equation:

$$
\begin{aligned}
& \mathrm{d} X(t)=\left[\pi_{t}(\mu+\lambda u(t)-r)+r X(t)\right] \mathrm{d} t \\
& +\pi_{t} \sigma P_{t}^{(1-q) / 2} \mathrm{~d} W(t)+\pi_{t} u(t) \mathrm{d} M(t) ; \\
& X(0)=x_{0} .
\end{aligned}
$$

Thus, the minimum-variance hedging problem can be written as

$$
\min _{\pi_{t}} \mathbb{E}[H-X(t)]^{2} ; \quad X(0)=x_{0} .
$$

\section{Optimal hedging portfolio}

In Sect. 2, we have formulated the minimum-variance hedging problem as a stochastic control problem. In this section, we will show how the backward stochastic differential equation method can be used to solve the stochastic control problem with jumps. Firstly, we introduce two lemmas on the existence of solutions of backward stochastic differential equations.

Lemma 1. The following backward stochastic differential equation

$$
\begin{gathered}
\mathrm{d} \alpha(t)=-\alpha(t)\left\{2 r-\frac{\left[\mu+\lambda u(t)-r+\frac{\sigma \beta(t) P^{(1-q) / 2}}{\alpha(t)}\right]^{2}}{\sigma^{2} P^{1-q}+\lambda u^{2}(t)}\right\} \mathrm{d} t \\
+\beta(t) \mathrm{d} W(t) ; \quad \alpha(T)=1,
\end{gathered}
$$

has a unique solution $(\alpha(t), \beta(t))$. Moreover, there exist two positive and finite constants $0<c_{1}<c_{2}<\infty$, such that $c_{1}<\alpha(t)<c_{2}, t \in[0, T]$. Finally, the stochastic differential equation

$$
\begin{aligned}
& \mathrm{d} h(t)=-\left[\frac{\sigma P^{(1-q) / 2}\left(\mu+\lambda u(t)-r+\frac{\sigma \beta(t) P^{(1-q) / 2}}{\alpha(t)}\right)}{\sigma^{2} P^{1-q}+\lambda u^{2}(t)}\right. \\
& \left.-\frac{\beta(t)}{\alpha(t)}\right] h(t) \mathrm{d} W(t) ; \quad h(0)=1
\end{aligned}
$$

also has a unique solution

$$
h(t)=\exp \left(-\frac{1}{2} \int_{0}^{t} \theta^{2}(s) \mathrm{d} s-\int_{0}^{t} \theta(s) \mathrm{d} W(s)\right),
$$

where $\theta(s)=\frac{\sigma P^{(1-q) / 2}\left(\mu+\lambda u(s)-r+\frac{\sigma \beta(s) P^{(1-q) / 2}}{\alpha(s)}\right)}{\sigma^{2} P^{1-q}+\lambda u^{2}(s)}-\frac{\beta(s)}{\alpha(s)}$. The solution $h(t)$ is a strictly positive square integrable martingale.

Proof of Lemma 1 It is not difficult to find that the existence and uniqueness of the solution $(\alpha(t), \beta(t))$ follows from the Theorem 5.1 in [30]. The existence of two positive and finite constants $0<c_{1}<c_{2}<\infty$, such that $c_{1}<h(t)<c_{2}, t \in[0, T]$ is shown in the proof of the same Theorem. The solution $h(t)$ is a strictly positive square integrable martingale following from the Theorem 4.1 of [30]. 
Lemma 2. The following backward stochastic differential equation:

$$
\begin{aligned}
& \mathrm{d} \varphi(t)=\left\{r \varphi(t)+\frac{\left[\mu+\lambda u(t)-r+\frac{\sigma \beta(t) P^{(1-q) / 2}}{\alpha(t)}\right]\left[\sigma \beta(t) P^{(1-q) / 2} \eta(t)+\lambda u(t) v(t)\right]}{\sigma^{2} P^{1-q}+\lambda u^{2}(t)}-\frac{\eta(t) \beta(t)}{\alpha(t)}\right\} \mathrm{d} t \\
& +\eta(t) \mathrm{d} W(t)+v(t) \mathrm{d} M(t) ; \quad \varphi(T)=H,
\end{aligned}
$$

has a unique solution $(\varphi(t), \eta(t), v(t))$.

\section{Proof of Lemma 2 Let}

$$
\theta(t)=\frac{\sigma P^{(1-q) / 2}\left(\mu+\lambda u(t)-r+\frac{\sigma \beta(t) P^{(1-q) / 2}}{\alpha(t)}\right)}{\sigma^{2} P^{1-q}+\lambda u^{2}(t)}-\frac{\beta(t)}{\alpha(t)}, \quad \gamma(t)=\frac{u(t)\left[\mu+\lambda u(t)-r+\frac{\sigma \beta(t) P^{(1-q) / 2}}{\alpha(t)}\right]}{\sigma^{2} P^{1-q}+\lambda u^{2}(t)} .
$$

Substituting (12) and (13) into (11), then, Eq. (11) becomes

$$
\mathrm{d} \varphi(t)=r \varphi(t) \mathrm{d} t+\eta(t)[\theta(t) \mathrm{d} t+\mathrm{d} W(t)]+v(t)[\lambda \mathrm{d} \gamma(t)+\mathrm{d} M(t)] ; \quad \varphi(T)=H .
$$

Now, we consider the following stochastic differential equation:

$$
\mathrm{d} f(t)=-f\left(t_{-}\right)[\theta(t) \mathrm{d} W(t)+\gamma(t) \mathrm{d} M(t)] ; \quad f(0)=1 .
$$

It is not difficult to obtain that the solution of (15) is

$$
f(t)=\xi_{1}(t) \xi_{2}(t),
$$

where

$$
\xi_{1}(t)=-\xi_{1}\left(t_{-}\right) \theta(t) \mathrm{d} W(t), \quad \xi_{1}(0)=1, \quad \xi_{2}(t)=-\xi_{2}\left(t_{-}\right) \gamma(t) \mathrm{d} M(t), \quad \xi_{2}(0)=1,
$$

thus, we can get the solutions of $\xi_{1}(t)$ and $\xi_{2}(t)$ as follows:

$$
\xi_{1}(t)=\exp \left(-\frac{1}{2} \int_{0}^{t} \theta^{2}(s) \mathrm{d} s-\int_{0}^{t} \theta(s) \mathrm{d} W(s)\right), \quad \xi_{2}(t)=\exp \left(\int_{0}^{t} \lambda \gamma(s) \mathrm{d} s\right) \Pi_{0<s \leq t}(1-\gamma(s) \Delta N(s)) .
$$

Now, we define a probability measure $\mathcal{Q}$ equivalent to $\mathcal{P}$ on $\left(\Omega, \mathcal{G}_{t}\right)$ as follows:

$$
\left.\frac{\mathrm{d} \mathcal{Q}}{\mathrm{d} \mathcal{P}}\right|_{\mathcal{G}_{t}}=f(t), \quad P-\text { a.s. (almost sure in probability). }
$$

Applying the Girsanov Theorem [31], we know that the process

$$
\widetilde{W}(t)=W(t)+\int_{0}^{t} \theta(s) \mathrm{d} s
$$

is a $\mathcal{G}$-Brownian motion under the probability measure $\mathcal{Q}$ and

$$
\widetilde{M}(t)=M(t)+\int_{0}^{t} \lambda \gamma(s) \mathrm{d} s=N(t)-\int_{0}^{t} \lambda(1-\gamma(s)) \mathrm{d} s
$$

is a $\mathcal{G}$-martingale under the probability measure $\mathcal{Q}$.

Let $\varphi(t)=B(t) \mathbb{E}_{\mathcal{Q}}\left[\frac{H}{B(T)} \mid \mathcal{G}_{t}\right]$, then $\frac{\varphi(t)}{B(t)}$ is a $\mathcal{G}$-martingale under the probability measure $\mathcal{Q}$. By the use of the martingale representation theory (see [32]), we can obtain that there are unique square integrable $\mathcal{G}$-predictable processes $\bar{\eta}(t)$ and $\bar{v}(t)$, which can make the square integrable $\mathcal{G}$-martingale $\frac{\varphi(t)}{B(t)}$ be written as

$$
\frac{\varphi(t)}{B(t)}=\mathbb{E}_{\mathcal{Q}}\left[\frac{H}{B(T)}\right]+\int_{0}^{t_{-}} \bar{\eta}(s) \mathrm{d} \widetilde{W}(s)+\int_{0}^{t_{-}} \bar{v}(s) \mathrm{d} \widetilde{M}(s) .
$$

Using the Ito formula, we have

$$
\mathrm{d} \varphi(t)=r \varphi(t) \mathrm{d} t+\eta(t) \mathrm{d} \widetilde{W}(t)+v(t) \mathrm{d} \widetilde{M}(t)] ; \quad \varphi(T)=H,
$$

where $\eta(t)=B(t) \bar{\eta}(t)$ and $v(t)=B(t) \bar{v}(t)$. Substituting (16) and (17) into (19), we change the probability measure from $\mathcal{Q}$ back to $\mathcal{P}$. Then, (19) becomes into (14). Hence, Eq. (11) has a unique solution $(\varphi(t), \eta(t), v(t))$.

Theorem 1. Suppose that the solution $(\varphi(t), \eta(t), v(t))$ of $(11)$ is square integrable. Then, the minimal variance hedging portfolio for (7) is

$$
\pi(t)=\frac{\sigma P^{(1-q) / 2} \eta(t)+\lambda u(t) v(t)+\left[\mu+\lambda u(t)-r+\frac{\sigma P^{(1-q) / 2} \beta(t)}{\alpha(t)}\right]\left(\varphi\left(t_{-}\right)-x\left(t_{-}\right)\right)}{\sigma^{2} P^{1-q}+\lambda u^{2}(t)}
$$

and the optimal cost is

$$
C^{*}=\alpha(0)(\varphi(0)-x(0))^{2}+\mathbb{E} \int_{0}^{t}\left\{\lambda v^{2}(t)+\eta^{2}(t)-\frac{\left[\sigma P^{(1-q) / 2} \eta(t)+\lambda u(t) v(t)\right]^{2}}{\sigma^{2} P^{1-q}+\lambda u^{2}(t)}\right\} \mathrm{d} t
$$


Proof of Theorem 1 Using the Ito formula, we have

$$
\begin{aligned}
& \mathrm{d}\left[\alpha(t)(\varphi(t)-x(t))^{2}\right]=\left\{(\varphi(t)-x(t))^{2}\left[-2 r \alpha(t)+\alpha(t) \frac{\left.\mu+\lambda u(t)-r+\frac{\sigma P^{(1-q) / 2} \beta(t)}{\alpha(t)}\right]+2 r \alpha(t)(\varphi(t)-x(t))^{2}}{\sigma^{2} P^{1-q}+\lambda u^{2}(t)}\right]+\frac{\left(\mu+\lambda u(t)-r+\frac{\sigma P^{(1-q) / 2} \beta(t)}{\alpha(t)}\right)\left(\sigma P^{(1-q) / 2} \eta(t)+\lambda u(t) v(t)\right)}{\sigma^{2} P^{1-q}+\lambda u^{2}(t)}-\frac{\eta(t) \beta(t)}{\alpha(t)}\right]+\lambda \alpha(t) v^{2}(t) \\
& \quad+2 \alpha(t)(\varphi(t)-x(t))] \\
& \quad+\alpha(t) \pi^{2}(t)\left(\sigma^{2} P^{1-q}+\lambda u^{2}(t)\right)-2 \alpha(t) \pi(t)\left[\sigma P^{(1-q) / 2} \eta(t)+\lambda u(t) v(t)+(\mu+\lambda u(t)-r)(\varphi(t)-x(t))\right] \\
& \left.\quad+2 \beta(t)(\varphi(t)-x(t))\left(\eta(t)-\sigma P^{(1-q) / 2} \pi(t)\right)\right\} \mathrm{d} t+\left[\beta(t)(\varphi(t)-x(t))^{2}+2 \alpha(t)(\varphi(t)-x(t))\right. \\
& \left.\quad \times\left(\eta(t)-\sigma P^{(1-q) / 2} \pi(t)\right)\right] \mathrm{d} W(t)+\left[2 \alpha(t)(\varphi(t)-x(t))(v(t)-u(t) \pi(t))+\alpha(t)(v(t)-u(t) \pi(t))^{2}\right] \mathrm{d} M(t) .
\end{aligned}
$$

Using the local martingale theory, we know that there is an increasing sequence of stopping times $\left\{\tau_{n}\right\} \uparrow T$ as $n \rightarrow \infty$ to make the above equation become

$$
\begin{aligned}
& \mathbb{E}\left[\alpha\left(T \wedge \tau_{n}\right)\left(\varphi\left(T \wedge \tau_{n}\right)-x\left(T \wedge \tau_{n}\right)\right)^{2}\right]=\alpha(0)(\varphi(0)-x(0))^{2}+\mathbb{E} \int_{0}^{T \wedge \tau_{n}} \alpha(t)\left\{\lambda v^{2}(t)+\eta^{2}(t)\right. \\
& \left.-\left[\frac{\left(\sigma P^{(1-q) / 2} \eta(t)+\lambda u(t) v(t)\right)^{2}}{\sigma^{2} P^{1-q}+\lambda u^{2}(t)}\right]\right\} \mathrm{d} t+\mathbb{E} \int_{0}^{T \wedge \tau_{n}} \alpha(t)\left(\sigma^{2} P^{1-q}+\lambda u^{2}(t)\right) \\
& \quad \times\left\{\pi(t)-\left[\frac{\sigma P^{(1-q) / 2} \eta(t)+\lambda u(t) v(t)+\left(\mu+\lambda u(t)-r+\frac{\sigma P^{(1-q) / 2} \beta(t)}{\alpha(t)}\right)\left(\varphi\left(t_{-}\right)-x\left(t_{-}\right)\right)}{\sigma^{2} P^{1-q}+\lambda u^{2}(t)}\right]\right\}^{2} \mathrm{~d} t .
\end{aligned}
$$

In the case that $\alpha(t)$ and $\varphi(t)$ are uniformly bounded and $\mathbb{E}\left[\sup _{0 \leq t \leq T}|x(t)|\right]<\infty$, applying the dominated convergence Theorem and the monotone convergence Theorem, we have

$$
\begin{aligned}
& \mathbb{E}\left[\alpha(T)(\varphi(T)-x(T))^{2}\right]=\alpha(0)(\varphi(0)-x(0))^{2}+\mathbb{E} \int_{0}^{t} \alpha(t)\left\{\lambda v^{2}(t)+\eta^{2}(t)-\frac{\left[\sigma P^{(1-q) / 2} \eta(t)+\lambda u(t) v(t)\right]^{2}}{\sigma^{2} P^{1-q}+\lambda u^{2}(t)} \mathrm{d} t\right. \\
+ & \mathbb{E} \int_{0}^{t} \alpha(t)\left(\sigma^{2} P^{1-q}+\lambda u^{2}(t)\right)\left\{\pi(t)-\frac{\sigma P^{(1-q) / 2} \eta(t)+\lambda u(t) v(t)+\left[\mu+\lambda u(t)-r+\frac{\sigma P^{(1-q) / 2} \beta(t)}{\alpha(t)}\right]\left(\varphi\left(t_{-}\right)-x\left(t_{-}\right)\right)}{\sigma^{2} P^{1-q}+\lambda u^{2}(t)}\right\} \mathrm{d} t
\end{aligned}
$$

Let $\alpha(T)=1$ and $\varphi(T)=H$. It is not difficult to find that the necessary and sufficient condition of the minimal variance hedging portfolio is

$$
\pi(t)-\frac{\sigma P^{(1-q) / 2} \eta(t)+\lambda u(t) v(t)+\left(\mu+\lambda u(t)-r+\frac{\sigma P^{(1-q) / 2} \beta(t)}{\alpha(t)}\right)\left(\varphi\left(t_{-}\right)-x\left(t_{-}\right)\right)}{\sigma^{2} P^{1-q}+\lambda u^{2}(t)}=0 .
$$

Thus, we can obtain the optimal hedging portfolio

$$
\pi(t)=\frac{\sigma P^{(1-q) / 2} \eta(t)+\lambda u(t) v(t)+\left(\mu+\lambda u(t)-r+\frac{\sigma P^{(1-q) / 2} \beta(t)}{\alpha(t)}\right)\left(\varphi\left(t_{-}\right)-x\left(t_{-}\right)\right)}{\sigma^{2} P^{1-q}+\lambda u^{2}(t)}
$$

and the optimal cost is

$$
C^{*}=\alpha(0)(\varphi(0)-x(0))^{2}+\mathbb{E} \int_{0}^{t}\left[\lambda v^{2}(t)+\eta^{2}(t)-\frac{\left(\sigma P^{(1-q) / 2} \eta(t)+\lambda u(t) v(t)\right)^{2}}{\sigma^{2} P^{1-q}+\lambda u^{2}(t)}\right] \mathrm{d} t .
$$

Corollary 1. When the Tsallis non-extensive index $q=1$, the model reduces to normal statistics and the minimal variance hedging portfolio for (7) is

$$
\pi(t)=\frac{\sigma \eta(t)+\lambda u(t) v(t)+\left(\mu+\lambda u(t)-r+\frac{\sigma \beta(t)}{\alpha(t)}\right)\left(\varphi\left(t_{-}\right)-x\left(t_{-}\right)\right)}{\sigma^{2}+\lambda u^{2}(t)} .
$$

and the optimal cost is 


$$
C^{*}=\alpha(0)(\varphi(0)-x(0))^{2}+\mathbb{E} \int_{0}^{t}\left\{\lambda v^{2}(t)+\eta^{2}(t)-\frac{(\sigma \eta(t)+\lambda u(t) v(t))^{2}}{\sigma^{2}+\lambda u^{2}(t)}\right\} \mathrm{d} t
$$

Note: Substituting $q=1$ into Theorem 1, then the result of Corollary 1 is obtained. Moreover, the normal statistics is actually included in our model as a special case.

\section{Summary}

Recently, to develop more accurate asset price models, much effort has been done. In this paper, we introduce a new asset price model based on the Tsallis statistical mechanics and Poisson jumps, which can describe the characteristics of long-run dependence of asset prices and the skewness of the distribution of returns.

Furthermore, we consider the dynamic minimumvariance hedging problem in this incomplete market. By the use of stochastic control theory, we obtain a closed-form solution for the minimum-variance hedging policy.

As future work we will study the pricing of options and optimal portfolio selection problems with other constraints, such as value-at-risk limit, under this asset price model.

\section{Acknowledgments}

We would like to thank the referees for helpful comments. We wish to acknowledge financial support from the National Natural Science Foundation of China (Project No. 11171221), the Natural Science Foundation of Anhui Province (Project No. 1508085QA04), the Natural Science Key Foundation of the Education Department of Anhui Province (Project No. KJ2016A742) and the Humanities and Social Sciences Key Foundation of the Education Department of Anhui Province (Project No. SK2015A563).

\section{References}

[1] F. Black, M. Scholes, J. Polit. Econ. 81, 637 (1973).

[2] R.C. Merton, Bell J. Econ. Manag. Sci. 4, 141 (1973).

[3] V. Gontis, J. Ruseckas, A. Kononovičius, Physica A Statist. Mech. Appl. 389, 100 (2010).

[4] P.P. Tan, D.U.A. Galagedera, E.A. Maharaj, Physica A Statist. Mech. Appl. 391, 2330 (2012).

[5] A. Sensoy, B.M. Tabak, Physica A Statist. Mech. Appl. 436, 147 (2015).

[6] G.M. Viswanathan, U.L. Fulco, M.L. Lyra, M. Serva, Physica A Statist. Mech. Appl. 329, 273 (2003).

[7] B. Podobnik, P.C. Ivanov, K. Biljakovic, D. Horvatic, H.E. Stanley, I. Grosse, Phys. Rev. E 72, 026121 (2005).
[8] J.J. Tseng, S.P. Li, Physica A Statist. Mech. Appl. 390, 1300 (2011).

[9] C. Tsallis, J. Statist. Phys. 52, 479 (1988).

[10] F. Michael, M.D. Johnson, Physica A Statist. Mech. Appl. 320, 525 (2003).

[11] R. Rak, S. Drożdż, J. Kwapień, Physica A Statist. Mech. Appl. 374, 315 (2007).

[12] S.M.D. Queiros, L. Moyano, J. Souza, C. Tsallis, Europ. Phys. J. B Condens. Matter Compl. Syst. 55, 161 (2007).

[13] S. Stavroyiannis, I. Makris, V. Nikolaidis, Int. Rev. Fin. Anal. 19, 19 (2010).

[14] J. Kwapień, S. Drożdż, Phys. Rep. 515, 115 (2012).

[15] L. Borland, Quantitat. Fin. 2, 415 (2002).

[16] L. Borland, Phys. Rev. Lett. 89, 098701 (2002).

[17] C.R. Harvey, A. Siddique, J. Fin. Quantitat. Anal. 34, 465 (1999).

[18] J. Chen, H. Hong, J.C. Stein, J. Fin. Econ. 61, 345 (2001).

[19] R.F. Engle, A.J. Patton, Quantitat. Fin. 1, 237 (2001).

[20] P. Jorion, Rev. Fin. Studies 1, 427 (1988).

[21] S.G. Kou, Manag. Sci. 48, 1086 (2002).

[22] A.E.B. Lim, Math. Operat. Res. 29, 132 (2004).

[23] A.E.B. Lim, X.Y. Zhou, Math. Operat. Res. 27, 101 (2002).

[24] C. Gourieroux, J.P. Laurent, H. Pham, Math. Fin. 8, 179 (1998).

[25] J.P. Laurent, H. Pham, Fin. Stochast. 3, 83 (1999).

[26] M. Schweizer, Ann. Probabil. 24, 206 (1996).

[27] C. Tsallis, D.J. Bukman, Phys. Rev. E 54, R2197 (1996).

[28] T.R. Bielecki, M. Rutkowski, Credit Risk: Modeling, Valuation and Hedging, Springer Science \& Business Media, Berlin 2002.

[29] K.F.C. Yiu, J. Liu, T.K. Siu, W.K. Ching, Automatica 46, 979 (2010).

[30] A.E.B. Lim, Math. Operat. Res. 29, 132 (2004).

[31] I.V. Girsanov, Theory Probabil. Appl. 5, 285 (1960).

[32] X.X. Xue, Martingale Representation for a Class of Processes with Independent Increments and Its Applications, Springer, Berlin 1992. 